\title{
The Bright Gamma\#Ray Burst of 2000 February 10: A Case Study of an Optically Dark Gamma\#Ray Burst
}

\section{Citation}

Piro, L., D. A. Frail, J. Gorosabel, G. Garmire, P. Soffitta, L. Amati, M. I. Andersen, et al. 2002.

"The Bright Gamma\#Ray Burst of 2000 February 10: A Case Study of an Optically Dark Gamma\# Ray Burst." The Astrophysical Journal 577 (2): 680-90. https://doi.org/10.1086/342226.

\section{Permanent link}

http://nrs.harvard.edu/urn-3:HUL.InstRepos:41417371

\section{Terms of Use}

This article was downloaded from Harvard University's DASH repository, and is made available under the terms and conditions applicable to Other Posted Material, as set forth at http:// nrs.harvard.edu/urn-3:HUL.InstRepos:dash.current.terms-of-use\#LAA

\section{Share Your Story}

The Harvard community has made this article openly available.

Please share how this access benefits you. Submit a story.

Accessibility 


\title{
The bright Gamma-Ray Burst of February 10, 2000: a case study of an optically dark GRB
}

\author{
L. Piro ${ }^{1}$, D. A. Frail ${ }^{2}$, J.Gorosabel ${ }^{3,4}$, G. Garmire ${ }^{5}$, P. Soffitta ${ }^{1}$, L. Amati ${ }^{6}$, M.I. Andersen ${ }^{7}$, \\ L. A. Antonelli ${ }^{8}$ E.Berger $^{9}$, F. Frontera ${ }^{6,10}$, J. Fynbo ${ }^{11}$, G. Gandolfi ${ }^{1}$, M.R. Garcia ${ }^{12}$, J. \\ Hjorth $^{13}$, J. in 't Zand ${ }^{14}$ B.L. Jensen ${ }^{13}$, N.Masetti ${ }^{6}$, P. Møller ${ }^{11}$, H. Pedersen ${ }^{13}$, E. Pian ${ }^{15}$, \\ M. H. Wieringa ${ }^{16}$
}

\begin{abstract}
The gamma-ray burst GRB 000210 had the highest $\gamma$-ray peak flux of any event localized by BeppoSAX as yet but it did not have a detected optical afterglow, despite prompt and deep searches down to $R_{\text {lim }} \approx 23.5$. It is therefore one of the events recently classified as dark GRBs, whose origin is still unclear.
\end{abstract}

\footnotetext{
${ }^{1}$ Istituto Astrofisica Spaziale \& Fisica Cosmica, C.N.R., Via Fosso del Cavaliere, 00133 Roma, Italy

${ }^{2}$ National Radio Astronomy Observatory, Socorro, NM, 87801, USA

${ }^{3}$ Danish Space Research Institute, Juliane Maries Vej 30, DK-2100 Copenhagen Ø, Denmark

${ }^{4}$ Instituto de Astrofísica de Andalucía, CSIC, Apdo. Correos 3004, 18080 Granada, Spain

${ }^{5}$ Department of Astronomy and Astrophysics, 525 Davey Lab, Penn State University, University Park, PA 16802, USA

${ }^{6}$ Istituto Astrofisica Spaziale \& Fisica Cosmica, sezione Bologna, C.N.R.,Via Gobetti 101, 40129 Bologna, Italy

${ }^{7}$ Division of Astronomy, University of Oulu P.O. Box 3000, FIN-90014 University of Oulu, Finland

${ }^{8}$ Osservatorio Astronomico Roma, INAF, Via Frascati 33, 00040 Monte Porzio Catone, Roma, Italy

${ }^{9}$ California Institute of Technology, Palomar Observatory 105-24, Pasadena, CA 91125

${ }^{10}$ Dip. Fisica, Universita' Ferrara, Via Paradiso 12, Ferrara, Italy

${ }^{11}$ European Southern Observatory, Karl-Schwarzschild-Straße 2, D-85748 Garching, Germany

${ }^{12}$ Harvard-Smithsonian Center for Astrophysics, 60 Garden St. Cambridge, MA 02138, USA

${ }^{13}$ Astronomical Observatory, University of Copenhagen, Juliane Maries Vej 30, DK-2100 Copenhagen $\varnothing$, Denmark

${ }^{14}$ Space Research Organization in the Netherlands, Sorbonnelaan 2, 3584 CA Utrecht, The Netherlands

${ }^{15}$ Osservatorio Astronomico Trieste, INAF, Via G. Tiepolo 11, I-34131 Trieste, Italy

${ }^{16}$ Paul Wild Observatory, Locked Bag 194, Narribri NSW 2390, Australia
} 
Chandra observations allowed us to localize the X-ray afterglow of GRB 000210 to within $\approx 1^{\prime \prime}$ and a radio transient was detected with the VLA. The precise $\mathrm{X}$-ray and radio positions allowed us to identify the likely host galaxy of this burst, and to measure its redshift, $z=0.846$. The probability that this galaxy is a field object is $\approx 1.6 \times 10^{-2}$. The X-ray spectrum of the afterglow shows significant absorption in excess of the Galactic one corresponding, at the redshift of the galaxy, to $N_{H}=(5 \pm 1) \times 10^{21} \mathrm{~cm}^{-2}$. The amount of dust needed to absorb the optical flux of this object is consistent with the above HI column density, given a dust-to-gas ratio similar to that of our Galaxy. We do not find evidence for a partially ionized absorber expected if the absorption takes place in a Giant Molecular Cloud. We therefore conclude that either the gas is local to the GRB, but is condensed in small-scale high-density $\left(n \gtrsim 10^{9} \mathrm{~cm}^{-3}\right)$ clouds, or that the GRB is located in a dusty, gas-rich region of the galaxy. Finally, we examine the hypothesis that GRB 000210 lies at $z \gtrsim 5$ (and therefore that the optical flux is extinguished by $\mathrm{Ly}_{\alpha}$ forest clouds), but we conclude that the X-ray absorbing medium would have to be substantially thicker from that observed in GRBs with optical afterglows.

Subject headings: cosmology: observations — gamma-rays: bursts

\section{Introduction}

It is observationally well-established that about half of accurately localized gamma-ray bursts (GRBs) do not produce a detectable optical afterglow (Frail et al. 2000; Fynbo et al. 2001 ), while most of them $(\approx 90 \%$ ) have an X-ray afterglow (Piro 2001). Statistical studies have shown that the optical searches of these events, known variously as "dark GRBs", "failed optical afterglows" (FOA), or "gamma-ray bursts hiding an optical source-transient" (GHOST), have been carried out to magnitude limits fainter on average than the known sample of optical afterglows (Lazzati et al. (2002); Reichart \& Yost (2001), but see also Fynbo et al. (2001)). Some of these GRBs could be intrinsically faint events, but this fraction cannot be very high, because the majority of dark GRBs shows the presence of an X-ray afterglow similar to that observed in GRBs with optical afterglows (Piro 2001; Lazzati et al. 2002). Thus dark bursts could constitute a distinct class of events and not only be the result of an inadequate optical search, but it is unclear whether this observational property derives from a single origin or it is a combination of different causes.

If the progenitors of long-duration GRBs are massive stars (Paczyński 1998), as current

evidence suggests (e.g., Bloom et al. (1999); Piro et al. (2000)), extinction of optical flux 
by dusty star-forming regions is likely to occur for a substantial fraction of events (the obscuration scenario). Another possibility is that dark GRBs are located at redshift $z \gtrsim 5$, with the optical flux being absorbed by the intervening Ly $\alpha$ forest clouds (the high-redshift scenario).

Dark bursts which can be localized to arcsecond accuracy, through a detection of either their X-ray or radio afterglow, are of particular interest. The first and best-studied example was GRB 970828 for which prompt, deep searches down to $\mathrm{R} \sim 24.5$ failed to detect an optical afterglow (Odewahn et al. 1997; Groot et al. 1998) despite it was localized within a region of only $10^{\prime \prime}$ radius by the ROSAT satellite (Greiner et al. 1997). Djorgovski et al. (2001) recently showed how the detection of a short-lived radio transient for GRB 970828 allowed them to identify the probable host galaxy and to infer its properties (redshift, luminosity and morphology). In addition, they used estimates of the column density of absorbing gas from X-ray data, and lower limits on the rest frame extinction $\left(\mathrm{A}_{V}>3.8\right)$ to quantify the amount of obscuration towards the GRB.

Given the extreme luminosity of GRBs and their probable association with massive stars, it is expected that some fraction of events will be located beyond $z>5$ (Lamb \& Reichart 2000). These would be probably classified as dark bursts because the UV light, which is strongly attenuated by absorption in the $\mathrm{Ly}_{\alpha}$ forest, is redshifted into the optical band. Fruchter (1999) first suggested such an explanation for the extreme red color of the optical/NIR emission for GRB 980329, although an alternative explanation based on $\mathrm{H}_{2}$ absorption in the GRB environment would imply a somewhat lower redshift (Draine 2000). In a recent paper Jaunsen et al. (2002) derive a photometric redshift $z \approx 3.5$. We note that the three redshifts determined or suggested so far for dark GRBs $(z=0.96$, GRB970828, Djorgovski et al. (2001); $z=1.3$, GRB990506, Taylor et al. (2000); Bloom et al. (2002); $z \approx 0.47$, GRB000214, Antonelli et al. (2000)) are in the range of those measured for most bright optical afterglows, but whether this applies to the majority of these events is still to be assessed. Particularly interesting in this respect is the case of the so-called X-ray flashes or X-ray rich GRBs discovered by BeppoSAX(Heise et al. 2001). In most of these events no optical counterpart has been found. The only tentative association claimed as yet is for the event of Oct.30, 2001, where a candidate host galaxy of magnitude $\mathrm{V} \approx 25$ has been found in the direction of the afterglow (Fruchter et al. 2002). We note, however, that the probability that this object is a foreground galaxy is not negligible $\left(P \approx 3 \times 10^{-2}\right.$, see e.g. eq.2 in Sect.2.3). The high-redshift scenario would naturally explain both the absence of optical counterpart and the high-energy spectrum, because the peak of the gamma-ray spectrum would be redshifted into the X-ray band.

If we are to use dark bursts to study obscured star formation in the universe (Djorgovski 
et al. 2001), we must first understand the source of the extinction. For those afterglows which are not at $z>5$ it is important to establish whether they are dark because of a dense circumburst medium (Reichart \& Yost 2001), or their optical emission is extinguished by line-of-sight absorption from the medium of the host galaxy. We can use the properties of the afterglow, its location within the host galaxy, and the properties of the host galaxy itself to address this question. In this paper, we report observations of the burst GRB 000210 which was discovered and localized by BeppoSAX. A Chandra observation of the BeppoSAX error box enabled us to localize the likely host galaxy of the event and to identify a short-lived radio-transient, further refining the position to sub-arcsec accuracy. From sensitive upper limits on the absence of an optical afterglow we estimate the amount of extinction by dust and from the X-ray spectrum the amount of absorbing gas. GRB 000210 appears to be the newest member of a small but growing group of well-localized dark bursts (Frail et al. 1999; Taylor et al. 2000; Djorgovski et al. 2001).

\section{Observations}

\subsection{Gamma-Ray and X-ray Observations}

The gamma-ray burst GRB 000210 was detected simultaneously by the BeppoSAX Gamma-Ray Burst Monitor (GRBM) and Wide Field Camera 1 (WFC) on 2000 February 10, 08:44:06 UT. As of now, this event is the brightest GRB detected simultaneously by the GRBM and WFC, with a peak flux $\mathrm{F}(40-700 \mathrm{keV})=(2.1 \pm 0.2) \times 10^{-5} \mathrm{erg} \mathrm{cm}^{-2} \mathrm{~s}^{-1}$, ranking in the top $1 \%$ of the BATSE catalog. In X-rays the event was also very bright, with a peak flux $\mathrm{F}(2-10 \mathrm{keV})=(1.5 \pm 0.2) \times 10^{-7} \mathrm{erg} \mathrm{cm}^{-2} \mathrm{~s}^{-1}$, ranking fourth after GRB 990712 (Frontera et al. 2001), GRB 011121(Piro et al., in preparation) and GRB 01022(in' t Zand et al. 2001). The gamma-ray light curve shows a single, FRED-like ${ }^{17}$ pulse (Fig. 1), with a duration of about $15 \mathrm{~s}$. The X-ray light curve shows a longer pulse, with a tail persisting for several tens of seconds. The fluence $(40-700 \mathrm{keV})=(6.1 \pm 0.2) \times 10^{-5} \mathrm{erg} \mathrm{cm}^{-2} \mathrm{ranks}$ GRB 000210 in the top 5 brightest GRBs seen by the GRBM and WFC, and in the top $3 \%$ of the BATSE bursts (Kippen et al. 2000). With an $\mathrm{F}(2-10 \mathrm{keV}) / \mathrm{F}(40-700 \mathrm{keV})=0.007$, GRB 000210 is one of the hardest GRBs detected by BeppoSAX (Feroci et al. 2001). The spectrum is also very hard in the X-ray band. Time resolved spectra of the WFC, fitted with a power law model $F=C e^{-\sigma N_{H}} E^{-\Gamma}$, give $\Gamma=(0.38 \pm 0.13)$ and $\Gamma=(0.82 \pm 0.12)$ in the rising and first decaying part of the peak, with the usual hard-to-soft evolution continuing in the subsequent parts, with $\Gamma=2.3 \pm 0.15$ for $18 s<t<80 s$ (Fig. 1). The absorption column

\footnotetext{
${ }^{17}$ Fast Rise Exponential Decay
} 
density is consistent with that in our Galaxy $\left(N_{H G}=2.5 \times 10^{20} \mathrm{~cm}^{-2}\right)$, with an upper limit $N_{H} \lesssim 2 \times 10^{22} \mathrm{~cm}^{-2}$.

The GRB was localized with the WFC at (epoch J2000) R.A. $=01^{\mathrm{h}} 59^{\mathrm{m}} 14.9^{\mathrm{s}}$, dec. $=$ $-40^{\circ} 40.14^{\prime}$ within a radius of $2^{\prime}$. This position is consistent with the IPN annulus derived by Ulysses, Konus and the BeppoSAX/GRBM (Hurley et al. 2000). Prompt dissemination of the coordinates (Gandolfi et al. 2000; Stornelli et al. 2000)) triggered follow-up observations by several ground-based and space observatories, including BeppoSAX and Chandra. A BeppoSAX target-of-opportunity observation (TOO) started on Feb. 10.66 UT (7.2 hrs after the GRB) and lasted until Feb. 11.98 UT. Net exposure times were 44 ks for the MECS and $15 \mathrm{ks}$ for the LECS. The X-ray fading afterglow was detected (Costa et al. 2000) within the WFC error circle at (epoch J2000) R.A. $=01^{\mathrm{h}} 59^{\mathrm{m}} 15.9^{\mathrm{s}}$, dec. $=-40^{\circ} 39^{\prime} 29^{\prime \prime}$ (error radius $=50^{\prime \prime}$, see Fig. 2). The X-ray flux from the source exhibited a decay consistent with the standard power-law behavior, with $\mathrm{F}(2-10 \mathrm{keV})=3.5 \times 10^{-13} \mathrm{erg}^{-2} \mathrm{~cm}^{-1}$ in the first $30 \mathrm{ks}$ of the observation (Fig. 3). The spectrum derived by integrating over the entire observation is well fitted by a power law with photon index $\Gamma=1.75 \pm 0.3$, column density $N_{H}<4 \times 10^{21} \mathrm{~cm}^{-2}$, consistent with that in our Galaxy and flux $\mathrm{F}(2-10 \mathrm{keV})=2.2 \times 10^{-13} \mathrm{erg} \mathrm{cm}^{-2} \mathrm{~s}^{-1}$.

The Chandra TOO started approximately 21 hrs after the burst (i.e., around the middle of the BeppoSAX TOO), with an exposure time of $10 \mathrm{ks}$ with ACIS-S with no gratings. The X-ray afterglow was near the center of the BeppoSAX NFI region (Fig. 2). The initial position (Garcia et al. 2000b) derived from the first data processing by the Chandra X-ray Center was found to be affected by an aspect error of about 8" (Garcia et al. 2000a). Reprocessing of the data with the correct attitude calibration solved this problem (Garmire et al. 2000). We have further improved this position, following the prescription suggested by the Chandra team ${ }^{18}$, using 5 stars detected in X-rays and comparing their positions to the USNO A2 and 2MASS catalog positions. The final position of the GRB is (epoch J2000) R.A. $=01^{\mathrm{h}} 59^{\mathrm{m}} 15.58^{\mathrm{s}}$, dec. $=-40^{\circ} 39^{\prime} 33.02^{\prime \prime}$, with an estimated error radius of $0^{\prime \prime} 6(90 \%$ confidence level).

The ACIS-S total counts were $555 \pm 26$, corresponding to $\mathrm{F}(2-10 \mathrm{keV})=1.8 \times 10^{-13} \mathrm{erg} \mathrm{cm}^{-2} \mathrm{~s}^{-1}$ for a best-fit power law ( $\chi^{2}=20.9$ with 22 d.o.f.) with index $\Gamma=2.0 \pm 0.2$ and a significant amount of absorption $N_{H}=(0.17 \pm 0.04) \times 10^{22} \mathrm{~cm}^{-2}$, well above that expected from our Galaxy. We have performed a simultaneous fit to the Chandra ACIS-S, and BeppoSAX MECS and LECS data with an absorbed power law, leaving the relative normalization of the instruments free, to account for the non-simultaneous coverage of the decaying source. The resulting fit (Fig. 4) is satisfactory $\left(\chi^{2} /\right.$ d.o.f. $\left.=26 / 34\right)$. In particular the ACIS-S/MECS

\footnotetext{
${ }^{18} \mathrm{http}: / /$ asc.harvard.edu/mta/ASPECT/
} 
relative normalization is $1.08 \pm 0.2$, and $\Gamma=1.95 \pm 0.15$. In the inset of Fig. 4 we present the confidence levels of the intrinsic hydrogen column density as a function of the redshift of the source.

In Fig. 3 we plot the light curve in the $2-10 \mathrm{keV}$ range from the prompt emission to the afterglow. Frontera et al. (2000) have argued that on average, the afterglow starts at a time $\approx 60 \%$ of the duration of the burst. This is consistent with what we observe in GRB 000210 . The steep gradient of the WFC light curve flattens out around $t=20 \mathrm{~s}$, suggesting that at this time the afterglow starts dominating over the prompt emission. In fact, the WFC data points at $t \gtrsim 20 s$ and the BeppoSAX and Chandra data follow a power law decay $\left(\mathrm{F} \propto t^{-\alpha_{x}}\right)$

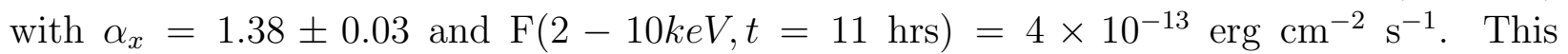
interpretation is also supported by the spectral behaviour, with the WFC spectral index attaining a value of $\Gamma=(2.3 \pm 0.15)$ around $\mathrm{t}=20 \mathrm{~s}$ consistent with that observed at later times by the BeppoSAX NFI and Chandra ACIS-S. There is no evidence for a break in the $\mathrm{X}$-ray light curve, which would have been clearly detected if present in such a bright burst (e.g. GRB 990510, Pian et al. (2001), GRB 010222, in' t Zand et al. (2001)).

\subsection{Optical Observations}

We obtained optical imaging of the field of GRB 000210 with the 2.56-m Nordic Optical Telescope (NOT) equipped with the HIRAC instrument and with the 1.54-m Danish Telescope (1.54D) plus DFOSC starting 12.4 hours and 16 hours after the burst, respectively. Further observations were carried out in August and in October 2000. A log of these observations can be found in Table 1, while in Table 2 we list the secondary standards used for the calibration of the optical magnitudes of the field.

The observations (Fig. 5) revealed a faint extended object located within the Chandra error circle (Gorosabel et al. 2000). The contours of the optical emission (Fig. 6) show that the source is slightly elongated in the North-East direction with an angular extension of $\sim 1^{\prime \prime}$.5. The angular size in the orthogonal direction (North-West) is limited by the seeing ( $0^{\prime \prime}$.7 in our best images). The center of the object (i.e. excluding the diffuse component) has coordinates (epoch 2000) R.A. $=01^{\mathrm{h}} 59^{\mathrm{m}} 15.61^{\mathrm{s}}$, dec. $=-40^{\circ} 39^{\prime} 33.1^{\prime \prime}$ with a $90 \%$ uncertainty of $0^{\prime \prime} 74$ which represents the sum in quadrature of a systematic error of 0.4 and a statistical

error of 0 ' 6 . This position is the mean value of astrometric calibrations on three independent images based on the USNO A2.0 catalog. The position of two of the stars detected in the Chandra image, that are included in the smaller field of view of the optical image, demonstrates that the optical and X-ray fields are tied to within $\approx 0.2(90 \%$ confidence level). A comparison of early and late observations shows that the object remains constant 
in brightness within the photometric errors, with magnitudes $\mathrm{B}=25.1 \pm 0.7, \mathrm{~V}=24.1 \pm 0.15$, $\mathrm{R}=23.5 \pm 0.1, \mathrm{I}=22.60 \pm 0.12$. Although the object in Fig. 6 is very faint, its appearance is not stellar.

\subsection{The host galaxy?}

Spectroscopic observations confirmed that the object is a galaxy. The observations were carried out on 2000 October 25 with the VLT1 equipped with FORS1. The 300V grism and the 0.7 slit provided a spectral FWHM resolution of $\sim 10 \AA$. The spectrum is based on a single 2000 sec exposure and covers the range between $4193 \AA$ and $8380 \AA$. The reduction is based on standard procedures, i.e., flat field correction with internal lamp flats and wavelength calibration using arc lamps. No spectro-photometric standard stars were observed, so no flux calibration was possible.

The spectrum revealed an emission line at $6881.2 \pm 0.5 \AA, \sim 6 \sigma$ above the continuum level (Fig. 7)). Given the presence of a well-detected continuum blueward of the line, and the absence of other emission lines in the $4193-8380 \AA$ range, we identify this line with the $3727 \AA[\mathrm{OII}]$ line at a redshift of $z=0.8463 \pm 0.0002$. The equivalent width of the line is $E W=(68 \pm 9) \AA$. We stress that the absence of $\mathrm{V}$ dropout in photometric data, expected by $\mathrm{Ly}_{\alpha}$ forest absorption at high $\mathrm{z}$, indicates by itself that $z<4$ (Madau 1995).

Is this galaxy the host of GRB 000210? We have computed the probability of a chance association in two ways. First we have computed the probability of finding an unrelated field galaxy with $R<R_{\text {host }}=23.5$ within the localization circle of the afterglow ( $1^{\prime \prime}$ radius, i.e. the $99 \%$ Chandra error radius). From galaxy counts (Hogg et al. 1997) we derive that $P=10^{-2}$. A slightly more conservative estimation is given by the the fraction of the sky covered by galaxies brighter than $R_{\text {host }}$ :

$$
P=\int^{R_{h o s t}} A(m) \frac{d N}{d m} d m
$$

where $d N / d m$ is the mean number of galaxies per magnitude per unit solid angle and $A(m)$ is the average area of a galaxy of R-band magnitude $m$. For $m>21 d N / d m \approx 10^{0.334 * m}$ (Hogg et al. 1997), while for brighter galaxies $d N / d m \approx 10^{0.5 * m}$ (Koo \& Kron 1992). We have estimated the average area of a galaxy $A=\pi\left(2 r_{h l}\right)^{2}$, where $r_{h l}$ is the half-light radius of the galaxy. We have adopted the following empirical half-light radius-magnitude relations: $r_{h l}=0^{\prime \prime} 6 \times 10^{-0.075 *(m-21)}$, for $21<m<27$ (Odewhan et al. 1996; Bloom et al. 2002), and $r_{h l}=0^{\prime \prime} 6 \times 10^{-0.2 *(m-21)}$ for brighter galaxies (Im et al. 1995, and references therein). By substituting these relations in eq.1, we derive: 


$$
P=\left[4.9+3.8 \times 10^{0.184\left(R_{\text {host }}-21\right)}\right] \times 10^{-3} \quad 21<R_{\text {host }}<27
$$

For $R_{\text {host }}=23.5, P=1.6 \times 10^{-2}$. Therefore, a chance association is unlikely, but not completely negligible.

\subsection{Radio Observations}

Very Large Array (VLA) ${ }^{19}$ observations were initiated within 15 hours after the burst. Details of this and all subsequent VLA observations are given in Table 3. In addition, a single observation was also made two days after the burst with the Australian Telescope Compact Array (ATCA). ${ }^{20}$ All VLA observations were performed in standard continuum mode, with a central frequency of $8.46 \mathrm{GHz}$, using the full $100 \mathrm{MHz}$ bandwidth obtained in two adjacent $50 \mathrm{MHz}$ bands. The flux density scale was tied to the extragalactic source 3C48 (J0137+331), while the phase was monitored using the nearby source J0155-408. The ATCA observation was made at a central frequency of $8.7 \mathrm{GHz}$, with a $256 \mathrm{MHz}$ bandwidth obtained in two adjacent $128 \mathrm{MHz}$ bands. The flux density scale was tied to the extragalactic source J1934-638, while the phase was monitored using the nearby source J0153-410.

On 2000 February 18.95 UT a radio source was detected within the Chandra error circle with a peak brightness of $99 \pm 21 \mu \mathrm{Jy}_{\text {beam }}{ }^{-1}$, or a flux density (after Gaussian fitting) of $93 \pm 21 \mu \mathrm{Jy}$. The synthesized beam was $6^{\prime \prime}$ by $2^{\prime \prime}$, with a position angle on the sky of $30^{\circ}$ counterclockwise. The position of this source is (epoch J2000) R.A. $=01^{\mathrm{h}} 59^{\mathrm{m}} 15.57^{\mathrm{s}}\left( \pm 0.05^{\mathrm{s}}\right)$, dec. $=-40^{\circ} 39^{\prime} 31.9^{\prime \prime}\left( \pm 1^{\prime \prime} .0\right)$, where the errors are at $90 \%$ confidence level in the Gaussian fit. We have further refined the astrometry, by looking for coincident USNO2 stars. We find one radio source coincident with a star, shifted by an offset of $(-0.05 \mathrm{~s}, 0$. 56$)$ in R.A. and dec. Taking into account this offset, we derive the final position of the radio counterpart of the GRB at R.A. $=01^{\mathrm{h}} 59^{\mathrm{m}} 15.62^{\mathrm{s}}$, dec. $=-40^{\circ} 39^{\prime} 32.46^{\prime \prime}$. The position of this radio transient is in excellent agreement with the X-ray afterglow and the optical source (Fig.6). The transient nature of this source is readily apparent (see Table 3), since it was not detected in observations either before or after February 18.

\footnotetext{
${ }^{19}$ The NRAO is a facility of the National Science Foundation operated under cooperative agreement by Associated Universities, Inc. NRAO operates the VLA.

${ }^{20}$ The Australia Telescope is funded by the Commonwealth of Australia for operation as a National Facility managed by CSIRO.
} 


\section{Discussion}

\subsection{Properties of the host}

In the following we will assume a cosmology with $\mathrm{H}_{\circ}=65 \mathrm{~km} \mathrm{~s}^{-1} \mathrm{Mpc}^{-1}, \Omega_{m}=0.3$, and $\Omega_{\Lambda}=0.7$. At $z=0.846$, the luminosity distance $D_{L}=1.79 \times 10^{28} \mathrm{~cm}$, and 1 arcsec corresponds to 8.3 proper $\mathrm{kpc}$ in projection. The $\gamma$-ray fluence implies an isotropic $\gamma$-ray energy release $E_{\gamma}=1.3 \times 10^{53}$ erg.

The [OII]3727 line flux can be used to estimate the star formation rate of the galaxy (Kennicutt 1998). Although the spectrum has not been calibrated, we have derived a rough estimation of the line flux by rescaling the line flux measured in the host galaxy of GRB 970828 for the line EW, R magnitudes and redshifts (Djorgovski et al. 2001). We find $L_{3727} \approx 1-2 \times 10^{41} \mathrm{erg} \mathrm{s}^{-1}$, corresponding to $\mathrm{SFR} \approx 3 M_{\odot} \mathrm{yr}^{-1}$. Since we are not sensitive to any obscured components of SFR, this relatively modest SFR is only a lower limit to the true star forming rate. From the I band photometry we derive a rest frame absolute magnitude $\mathrm{M}_{B}=-19.9 \pm 0.1$, corresponding to a $\approx 0.5 L_{*}$ galaxy today (Schechter 1976). The location of the afterglow is within $\approx 1^{\prime \prime}$ the center of the galaxy, corresponding to about $8 \mathrm{kpc}$ in projection.

\subsection{The nature of the obscuring medium}

The Chandra and BeppoSAX observations detected an X-ray afterglow which clearly underwent a power-law decay with $\alpha_{x}=1.38 \pm 0.03$. There is no evidence for a temporal break in the X-ray light curve between $10 \mathrm{~s}$ and $2 \times 10^{5} \mathrm{~s}$ after the burst (Fig. 3). A deviation from a power law decay could occur if the synchrotron cooling break $\nu_{c}$ passed through the band, or the outflow began to exhibit jet geometry, or at the transition to non-relativistic expansion. In all respects the X-ray afterglow of GRB 000210 was fairly typical in comparison with past events (Piro 2001).

The VLA and ATCA measurements indicate that the only significant detection of the radio afterglow from GRB 000210 was nine days after the burst. With a $8.46 \mathrm{GHz}$ flux density of $93 \pm 21 \mu \mathrm{Jy}$, this is the weakest radio afterglow detected to date. The time interval between the burst and the radio peak is too long to be the result of a reverse shock propagating in the relativistic ejecta (Kulkarni et al. 1999; Sari \& Piran 1999). It is more likely that the emission originated from a forward shock which reached a maximum on this timescale (e.g. Frail et al. 1999). Interstellar scintillation can briefly increase (or decrease) the radio flux of a weak afterglow and make it detectable (Goodman 1997; Waxman et al. 1998). With 
these single-frequency measurements it cannot be determined whether the $8.46 \mathrm{GHz}$ flux density was weak because GRB 000210 was a low energy event, or because the synchrotron self-absorption frequency was high $\left(\nu_{a b}>10 \mathrm{GHz}\right)$. Reichart \& Yost (2001) have argued that a high $\nu_{a b}$ is expected for dark bursts if they occur in dense circumburst environments $\left(n>>10^{2} \mathrm{~cm}^{-3}\right)$.

Although the X-ray and radio afterglow were detected for GRB 000210, there are only lower limits for the magnitude of the expected optical transient of $\mathrm{R}>22$ and $\mathrm{R}>23.5$ at $12.4 \mathrm{hrs}$ and $16 \mathrm{hrs}$ after the burst, respectively. Following Djorgovski et al. (2001) we can use the fireball model to predict the expected optical flux and then derive a lower limit to the amount of the extinction. The simplest model of an isotropic fireball expanding into a constant density medium is assumed (Sari et al. 1998). This assumption is well justified since all well studied afterglows are better explained by expansion in a constant density medium rather than in a wind-shaped one (Panaitescu \& Kumar 2001). For the timescales of interest $(t<21 \mathrm{hrs})$, there is no evidence for a break in the X-ray light curve that would indicate a jet-like geometry or transition to non-relativistic expansion. Two limiting cases are considered. The first is when the cooling frequency $\nu_{c}$ lies below the optical band $\nu_{o}$ (i.e., $\nu_{c}<\nu_{o}$ ). The expected optical spectral flux density is $f_{o}=f_{x}\left(\nu_{o} / \nu_{x}\right)^{-p / 2}$, where $f_{x}$ is the X-ray spectral flux density at $\nu_{x}$. The second case is where the cooling frequency lies between the optical and X-ray bands (i.e., $\nu_{o}<\nu_{c}<\nu_{x}$ ). There is a local minimum when $\nu_{x} \simeq \nu_{c}$ and consequently the expected optical spectral density is $f_{o}=f_{x}\left(\nu_{o} / \nu_{x}\right)^{-(p-1) / 2}$. As long as $\nu_{c} \leq \nu_{x}$, the electron energy spectral index $\mathrm{p}=\left(2 / 3+4 / 3 \alpha_{x}\right)=2.51 \pm 0.04$. This value of $\mathrm{p}$ is also consistent with the spectral slope measured in X-rays.

The X-ray flux measured by Chandra 21 hrs after the burst was $\mathrm{F}(2-10 \mathrm{keV})=1.8 \times 10^{-13}$ erg $\mathrm{cm}^{-2} \mathrm{~s}^{-1}$, which corresponds to a spectral flux density $f_{x}=0.01 \mu \mathrm{Jy}$ at $4 \mathrm{keV}$ (adopting $\beta_{x}=(\Gamma-1)=0.95 \pm 0.15$ where $\left.\mathrm{f}_{x} \propto \nu^{-\beta_{x}}\right)$. For $\nu_{c}<\nu_{o}$ the predicted optical magnitudes are therefore $\mathrm{R} \sim 17.4$ and $\mathrm{R} \sim 17.7$ at $12.4 \mathrm{hrs}$ and $16 \mathrm{hrs}$ after the burst, respectively. The equivalent magnitudes for the case $\nu_{o}<\nu_{c} \simeq \nu_{x}$ are $\mathrm{R} \sim 21.5$ and $\mathrm{R} \sim 21.9$. Taking the more stringent limits on the absence of an optical transient 21 hrs after the burst, we infer significant extinction towards GRB 000210, with a range of upper limits lying between $\mathrm{A}_{\mathrm{R}}=1.6$ and $5.8 \mathrm{mag}$. Using the extinction curve as formulated by Reichart (2001), we convert these $A_{R}$ limits from the observer frame to a rest frame extinction of $A_{V}=0.9$ to 3.2 (for $z=0.85$ ), and derive a hydrogen equivalent column density $N_{H O} \gtrsim 0.2-0.6 \times 10^{22}$ $\mathrm{cm}^{-2}$ assuming the Galactic relation of Predehl \& Schmitt (1995).

How does this result compare with the estimate of absorption derived from the X-ray data? In Fig. 4 we show the contour plot of the X-ray column density in the GRB frame as a function of the redshift, under the assumption that the absorbing material is in a neutral 
cold state. At $z=0.85$ the absorption is $N_{H X}=(0.5 \pm 0.1) \times 10^{22} \mathrm{~cm}^{-2}$. Thus $N_{H X} / N_{H O}$ is consistent with unity, or less, and therefore the dust-to-gas ratio is compatible with that of our Galaxy, as it has also been found in the other dark GRB 970828 (Djorgovski et al. 2001). In contrast, Galama \& Wijers (2000) noted that a number of bursts with optical afterglows seem to exhibit large column density as inferred from X-ray afterglow data, but with little or no optical extinction, suggesting that GRBs destroy dust grains along the line of sight. Different authors (Waxman \& Draine 2000; Fruchter et al. 2001; Reichart 2001) present mechanisms by which dust in the circumburst medium is destroyed or depleted by the light from the optical flash and X-rays from the burst and early-time afterglow up to

a distance $R \approx 10 L_{49}^{1 / 2} \mathrm{pc}\left(\mathrm{L}=10^{49} L_{49} \mathrm{erg} \mathrm{s}^{-1}\right.$ is the isotropic-equivalent luminosity of the optical flash). Reichart (2001) and Reichart \& Price (2001) proposed a unified scenario to explain the two populations of GRBs. They argue that most GRB occur in giant molecular clouds (GMC), with properties similar to those observed in our Galaxy (size $\approx 20-90 \mathrm{pc}$, Solomon et al. (1987)). Assuming that the energy reservoir is standard (Frail et al. 2001; Piran et al. 2001), strongly collimated bursts would burn out completely through the clouds, thus producing a detectable optical afterglow regardless of the column density through the cloud. Weakly collimated bursts would not destroy all the dust, leaving a residual column density through the line of sight. If this column density is sufficiently high, optical photons of the afterglow will be extinguished, making a dark GRB.

We find, however, that this scenario is not consistent with the observed properties of the X-ray absorber, if GRB 000210 lies at $\mathrm{z}=0.846$. For typical densities of a GMC $\left(n \approx 10^{2}-10^{5} \mathrm{~cm}^{-3}\right.$ ), the gas should be ionized by GRB photons on scales of several parsecs (Boettcher et al. 1999; Lazzati \& Perna 2002). Over the whole cloud, the medium would span a wide range of ionization stages, from fully ionized to neutral, with a substantial fraction of the gas being in partially ionized stages. We have verified this case by fitting the BeppoSAX and Chandra spectra with an ionized absorber (model absori in XSPEC) at redshift $z=0.85$. The ionization stage is described by the ionization parameter $\xi=L_{X} / n R^{2}$, where $\mathrm{R}$ is the distance of the gas from the GRB. We do not find any evidence of an ionized absorber, with a tight upper limit of $\xi<1$. In fact, even a moderate level of ionization would result in a reduced opacity below $\approx 1 \mathrm{keV}$, due to the ionization of light elements such as $\mathrm{C}$ and $\mathrm{O}$, but this feature is not observed in the X-ray spectrum.

\subsubsection{High density clouds}

We have shown above that in the GMC scenario the X-ray absorbing gas is expected to be substantially ionized, contrary to what is observed. We now introduce a variation of 
the local absorption scenario, in which a phase of the medium is condensed in high density clouds or filaments with low filling factor. We show that this scenario is consistent with the properties of the X-ray absorption.

To keep the gas in a low-ionized stage at a distance of the order of few pc, a density $n \gtrsim 10^{9} \mathrm{~cm}^{-3}$ is required. In fact, the recombination time scale $t_{\text {rec }} \approx n^{-1} T^{-0.5}$, where $T$ is the electron temperature. In the case of iron, $t_{r e c}=300 n_{9}^{-1} T_{7}^{-0.5} s$ ( i.e. Piro et al. (2000); hereafter, given a quantity $X$, we define $X_{n}=10^{-n} X$ ). The typical temperature is expected to be in the range $T_{7} \approx 0.1-1$ (Piro et al. 2000; Paerels et al. 2000). Therefore, at sufficiently high density, recombination is effective in keeping the gas close to ionization equilibrium over timescales $\gtrsim t_{r e c}$, i.e., during the afterglow phase. The ionization parameter is then $\xi=\frac{L_{45}}{n_{9} R_{11}^{2}}$, where $L_{45}=3$ is the luminosity of the X-ray afterglow in the rest frame energy range $0.013-100 \mathrm{keV}$. For $n_{9} \gtrsim 1$ the medium is then in the neutral phase for distances $R_{18} \gtrsim 1$, as required. It is straightforward to show that this medium should be clumpy. The size of each clump has to be $r_{s} \lesssim N_{H} / n \lesssim 5 \times 10^{12} / n_{9} \mathrm{~cm}$, and the fraction of volume occupied by this medium (i.e., the filling factor) $f_{V}=N_{H} /(n R)=\frac{5 \times 10^{-6}}{n_{9} R_{18}}$. Finally we note that the size of a clump is much smaller than the zone of the fireball visible at the time of the observation, $\approx 10^{15} / \Gamma_{b}$, where $\Gamma_{b} \approx 2-10$ is the Lorentz factor of the fireball in the afterglow phase. We therefore require that most of the source is covered by these clouds, i.e., that the covering fraction $f_{\text {cov }}=f_{V} \frac{R}{r_{c}} \approx 1$. This condition is satisfied when $r_{s} \approx 5 \times 10^{12} / n_{9}$ $\mathrm{cm}$. The total mass contained in these clouds is $M_{c} \approx 3 R_{18}^{3} f_{V,-6} n_{9} M_{\odot}$.

It is well known from observations and models (Balsara et al. 2001; Ward-Thompson et al. 1994), that the medium in star-forming molecular clouds is clumpy, with dense clouds or filaments embedded in a much less dense intercloud medium. The largest densities are of the order of $\approx 10^{7} \mathrm{~cm}^{-3}$ (Nummelin et al. 2000), i.e. lower than required. However, both observations and modeling are limited in resolution to structures of size $\gtrsim 10^{16} \mathrm{~cm}$ and would then miss smaller and higher density fluctuations. Furthermore, the total mass of the X-ray absorbing clouds is a tiny fraction $\left(\approx 10^{-4}\right)$ of the mass of a GMC $\left(\approx 10^{5-6} M_{\odot}\right)$. Prima facie those structures could then be the tail in the power spectrum of density fluctuations in star forming regions. Interestingly, Lamb (2001) has also stressed the role of a dusty clumpy medium in GMC with regard to optical properties of GRBs. A more detailed discussion is beyond the scope of this paper.

It is worth noting that the density of this medium is similar to that of the gas responsible for iron features (e.g. Piro et al. 1999, 2000; Amati et al. 2000). This gas lies much closer to the burst and is therefore highly ionized. Interestingly, in the afterglow of GRB000210, we find a very marginal evidence of a recombination edge by H-like Fe atoms (at a confidence level of $97 \%$ ). The rest-frame energy of this feature is at $9.28 \mathrm{keV}$, corresponding, at $z=0.85$, 
to $\mathrm{E}=5 \mathrm{keV}$, where BeppoSAX data show some residual (Fig. 4). The $E W=(1 \pm 0.7) \mathrm{keV}$, is similar to that observed in GRB 991216 (Piro et al. 2000) and in the other dark GRB 970828 (Yoshida et al. 2001). This feature is the result of electron recombination on H-like Fe atoms and should be accompanied by a $\mathrm{K} \alpha$ line at $6.9 \mathrm{keV}$ with a similar intensity (Piro et al. 2000). Within the errors, the upper limit $E W<0.5 \mathrm{keV}$ to the latter line is consistent with this prediction.

\subsubsection{ISM absorption in the host galaxy}

We now discuss the hypothesis that the absorption does not take place in the circumburst environment of the GRB, but in the ISM of the host galaxy. We find that this scenario can easily account for the properties of this burst. In fact, it immediately explains the absence of ionization features in the X-ray absorber and a dust-to-gas ratio consistent with that in our Galaxy. The typical column density through a GMC is about $10^{22} \mathrm{~cm}^{-2}$ (Solomon et al. 1987), and therefore even a single GMC in the line of sight could provide the necessary absorption both in the optical and in X-rays. This scenario is also consistent with the location of the GRB, which lies within $\approx 10 \mathrm{kpc}$ from the center of the galaxy.

Could non-local ISM absorption by the host galaxy be the unique origin of the whole population of dark GRBs? Let us first assume that GRBs occur in the disk of a galaxy similar to ours at a typical distance of $10 \mathrm{kpc}$ from the center. This assumption is consistent with the visible properties of GRB host galaxies and the distribution of GRB offsets with respect to the host centre (Bloom et al. 2002). The line-of-sight column density of interstellar hydrogen gas measured from our location in the Galaxy (10 kpc from the center) is consistent with that required to make a $\operatorname{GRB} \operatorname{dark}\left(N_{H} \gtrsim 5 \times 10^{21} \mathrm{~cm}^{-2}\right)$ in a belt of about $\pm 5^{\circ}$ along the plane of the Galaxy (Dickey \& Lockman 1990). Thus, of the entire population of GRBs occurring in the disk of galaxies randomly oriented in the sky, only $\lesssim 10 \%$ would be dark, much below the observed fraction of $50-60 \%$. Therefore, while this hypothesis can account for a fraction of dark GRB's, including this one, in order to make up the entire population of dark events, GRB host galaxies should contain quantities of dust and gas, associated with obscured star-forming regions, much larger than a typical galaxy like ours.

\subsubsection{Implications for the absorber in the high-z scenario}

Finally, given the modest a posteriori probability, we have to consider the possibility that the association of GRB 000210 with the galaxy is coincidental, and that this GRB is 
located at $z \gtrsim 5$. In this case the X-ray absorber of GRB 000210 is thicker and it can also be much more ionized. For example, at $z=5, \xi \lesssim 10^{3}$, i.e., the data are consistent with an absorber from neutral to highly ionized. In particular we have satisfactorily fitted the data either with a neutral absorber $(\xi=0)$, that requires $N_{H}=9 \times 10^{22} \mathrm{~cm}^{-2}$, or an ionized absorber $(\xi=400)$, that gives $N_{H}=16 \times 10^{22} \mathrm{~cm}^{-2}$. Such values of column densities are much higher than those observed in X-ray afterglows of GRBs with optical transients (e.g. Piro et al. 2001; in' t Zand et al. 2001).

\section{Conclusions}

In this paper we have presented the results of multi-wavelength observations of GRB 000210. This event was the brightest ever observed in $\gamma$-rays in the BeppoSAX GRBM and WFC. Nonetheless, no optical counterpart was found down to a limit of $R=23.5$. GRB 000210 is therefore one of the events classified as dark GRBs, a class that makes up $\approx 50 \%$ of all GRBs. It is still unclear whether this class derives from a single origin or it is due to a combination of different causes. Some of these GRBs could be intrinsically faint events, but this fraction cannot be very high, because the majority of dark GRBs shows the presence of an X-ray afterglow similar to that observed in GRBs with optical afterglows (Piro 2001; Lazzati et al. 2002). The most compelling hypotheses to explain the origin of dark bursts involve absorption, occurring either in the local environment of the GRB (circumburst or interstellar), or as Ly $\alpha$ forest absorption for those bursts which have $z \gtrsim 5$.

As in the majority of bursts, GRB 000210 had an X-ray afterglow which was observed with BeppoSAX and Chandra. The temporal behavior is well described by a power law, with a decay index $\alpha_{x}=-1.38 \pm 0.03$, similar to that observed in several other events (e.g. Piro 2001). We did not find evidence for breaks in the light curve. The spectral index of the power law is also typical $(\Gamma=1.95 \pm 0.15)$.

Thanks to the arcsecond localization provided by Chandra we identified the likely host galaxy of this burst, determined its redshift $(z=0.846)$ and detected a radio afterglow. The properties of the X-ray afterglow allowed us to determine the amount of dust obscuration required to make the optical afterglow undetectable $\left(A_{R} \gtrsim 2\right)$. The X-ray spectrum shows significant evidence of absorption by neutral gas $\left(N_{H X}=(0.5 \pm 0.1) \times 10^{22} \mathrm{~cm}^{-2}\right)$. However, we do not find evidence of a partially ionized absorber expected if the absorption takes place in a Giant Molecular Cloud, as recently suggested to explain the properties of the dark GRBs (e.g. Reichart \& Yost 2001). We conclude that, if the gas is local to the GRB, it has to be condensed in dense $\left(n \gtrsim 10^{9} \mathrm{~cm}^{-3}\right)$ clouds. We propose that these clouds represent the small-scale high-density fluctuations of the clumpy medium of star-forming GMCs. 
Both the amount of dust required to extinguish the optical flux and the dust-to-gas ratio are consistent with those observed across the plane of our Galaxy. We cannot therefore exclude that the absorption takes place in the line-of-sight through the interstellar medium of the host, rather than being produced by a GMC embedding the burst. This hypothesis is also consistent with the location of GRB 000210 with respect to the center of the likely host galaxy. To explain the whole population of dark GRB this hypothesis would require that host galaxies of GRBs should be characterized by quantities of dust and gas much larger than typical, arguing again for a physical connection between GRBs and star forming regions.

Finally, we discussed the possibility that the galaxy is unrelated to GRB 000210 and that it is a dark burst because it lies at $z \gtrsim 5$. In this case the X-ray absorbing medium should be substantially thicker than that observed in GRBs with optical afterglows. Assuming that GRB 000210 is a typical representative of a population of events at high redshift, then these GRB's are embedded in a much denser environment than that of closer events.

Whichever of the explanations apply, it is clear that dark GRBs provide a powerful tool to probe their formation sites and possibly to explore the process of star formation in the Universe. We have at hand several observational tools to pursue this investigation. By increasing the number of arcsecond locations by radio, X-ray and far-infrared observations we can build up a sample of host galaxies of dark GRBs and study their distances and physical properties. The origin of the absorption in X-rays and optical can be addressed by broad band spectra and modeling, providing information on the dust and gas content of the absorbing structures. X-ray measurements are particularly promising in this respect for several reasons. First, X-rays do not suffer from absorption, in fact roughly the same number of dark GRB and GRBs with optical afterglows have an X-ray afterglow. Detection of X-ray lines can thus provide a direct measurement of the redshift. A comparative study of the $\mathrm{X}$ ray properties of these two classes should also underline differences that can be linked to their origin, like the brightness of X-ray afterglows and the amount of X-ray absorption. Finally, measurements of variability of the X-ray absorbing gas would provide strong support to the local absorption scenario. There are several mechanisms that can produce such a variability. The hard photon flux from the GRB and its afterglow will ionize the circumburst gas on short time scales, thus decreasing the effective optical depth with time (Perna \& Loeb 1998). The detection of a transient iron edge in GRB990705 (Amati et al. 2000) and the decrease of the column density from the prompt to the afterglow phases in GRB980329 (Frontera et al. 2000) and GRB010222 (in' t Zand et al. 2001) are both consistent with this scenario. The variable size of the observable fireball that increases with the inverse of the bulk Lorentz factor can also produce variations of the column density, if the medium surrounding the source is not homogeneous. In this regard, two X-ray afterglows show some, admittedly marginal, evidence of variability of $N_{H}$ (Piro et al. 1999; Yoshida et al. 2001). In conclusion, 
the future of the investigations of dark GRBs looks particularly bright.

BeppoSAX is a program of the Italian space agency (ASI) with participation of the Dutch space agency (NIVR). We would like to thank H. Tananbaum and the Chandra team as well as E. Costa, M. Feroci, J. Heise and the other members of the BeppoSAX team for the support in performing the observations with these satellites and an anonymous referee for useful suggestions. GG acknowledge support under NASA grant G00-1010X. MRG acknowledges support under NASA contract NAG8-39073 to the Chandra X-Ray Center. Part of the optical observations are based on observations made with the Danish 1.54-m telescope at ESO, La Silla, Chile. This research was supported by the Danish Natural Science Research Council through its Centre for Ground-based Observational Astronomy. Based in part on observations made with the Nordic Optical Telescope, operated on the island of La Palma jointly by Denmark, Finland, Iceland, Norway, and Sweden, in the Spanish Observatorio del Roque de los Muchachos of the Instituto de Astrofísica de Canarias. Some of the data presented here have been taken using ALFOSC, which is owned by the Instituto de Astrofísica de Andalucía (IAA) and operated at the Nordic Optical Telescope under agreement between IAA and the NBIfAFG of the Astronomical Observatory of Copenhagen. Partially based on ESO VLT programme 66.A-0386(A), Cerro Paranal, Chile.

\section{REFERENCES}

Amati, L., Frontera, F., Vietri, M., in’t Zand, J. J. M., Soffitta, P., Costa, E., del Sordo, S., Pian, E., Piro, L., Antonelli, L. A., dal Fiume, D., Feroci, M., Gandolfi, G., Guidorzi, C., Heise, J., Kuulkers, E., Masetti, N., Montanari, E., Nicastro, L., Orlandini, M., \& Palazzi, E. 2000, Science, 290, 953

Antonelli, L. A., Piro, L., Vietri, M., Costa, E., Soffitta, P., Feroci, M., Amati, L., Frontera, F., Pian, E., in 't Zand, J. J., Heise, J., Kuulkers, E., Nicastro, L., Butler, R. C., Stella, L., \& Perola, G. C. 2000, ApJ, 545, L39

Balsara, D., Ward-Thompson, D., \& Crutcher, R. M. 2001, MNRAS, 327, 715

Bloom, J. S., Kulkarni, S. R., \& Djorgovski, S. G. 2002, AJ, 123, 1111

Bloom, J. S. et al. 1999, Nature, 401, 453

Boettcher, M., Dermer, C. D., Crider, A. W., \& Liang, E. . P. 1999, A\&A, 343, 111

Costa, E. et al. 2000, GCN notice 542 
Dickey, J. M. \& Lockman, F. J. 1990, Ann. Rev. Astr. Ap., 28, 215

Djorgovski, S. G., , Frail, D. A., Kulkarni, S. R., Bloom, J. S., Odewahn, S. C., \& Dierks, A. 2001, ApJ, 562, 654

Draine, B. T. 2000, ApJ, 532, 273

Feroci, M., Antonelli, L. A., Soffitta, P., in 't Zand, J. J., Amati, L., Costa, E., Piro, L., Frontera, F., Pian, E., Heise, J., \& Nicastro, L. 2001, A\&A, 378, 441

Frail, D. A., Kulkarni, S. R., Bloom, J. S., Djorgovski, S. G., Gorjian, V., Gal, R. R., Meltzer, J., Sari, R., Chaffee, F. H., Goodrich, R., Frontera, F., \& Costa, E. 1999, ApJ, 525, L81

Frail, D. A., Kulkarni, S. R., Wieringa, M. H., Taylor, G. B., Moriarty-Schieven, G. H., Shepherd, D. S., Wark, R. M., Subrahmanyan, R., McConnell, D., \& Cunningham, S. J. 2000, in AIP Conf. Proc. 526: Gamma-ray Bursts, 5th Huntsville Symposium, 298-302

Frail, D. A. et al. 2001, ApJ, 562, L55

Frontera, F., Amati, L., Costa, E., Muller, J. M., Pian, E., Piro, L., Soffitta, P., Tavani, M., Castro-Tirado, A., Dal Fiume, D., Feroci, M., Heise, J., Masetti, N., Nicastro, L., Orlandini, M., Palazzi, E., \& Sari, R. 2000, ApJS, 127, 59

Frontera, F., Amati, L. Vietri, M., in 't Zand, J. J. M., , Costa, E., , Feroci, M., Heise, J., Masetti, N., Nicastro, L., Orlandini, M., Palazzi, E., Pian, E., Piro, L., \& Soffitta, P. 2001, ApJ, 550, L47

Fruchter, A. et al. 2002, GCN notice 1268

Fruchter, A. S. 1999, ApJ, 512, L1

Fruchter, A. S., Krolik, J. H., \& Rhoads, J. E. 2001, ApJ, 563, 597

Fynbo, J. U., Jensen, B. L., Gorosabel, J., Hjorth, J., Pedersen, H., Møller, P., Abbott, T., Castro-Tirado, A. J., Delgado, D., Greiner, J., Henden, A., Magazzù, A., Masetti, N., Merlino, S., Masegosa, J., Østensen, R., Palazzi, E., Pian, E., Schwarz, H. E., Cline, T., Guidorzi, C., Goldsten, J., Hurley, K., Mazets, E., McClanahan, T., Montanari, E., Starr, R., \& Trombka, J. 2001, A\&A, 369, 373

Galama, T. J. \& Wijers, R. A. M. J. 2001, ApJ, 549, L209

Gandolfi, G. et al. 2000, GCN notice 538 \& 539 
Garcia, M., Garmire, G., \& Piro, L. 2000a, GCN notice 548

Garcia, M., Piro, L., Garmire, G., \& Nichols, J. 2000b, GCN notice 544

Garmire, G., Piro, L., Stratta, G., Garcia, M., \& Nichols, J. 2000, GCN notice 782

Goodman, J. 1997, New Astr., 2, 449

Gorosabel, J. et al. 2000, GCN notice 545

Greiner, J., Schwarz, R., Englhauser, J., Groot, P. J., \& Galama, T. J. 1997, IAU Circ, 6757

Groot, P. J., Galama, T. J., Van Paradijs, J., Kouveliotou, C., Wijers, R. A. M. J., Bloom, J., Tanvir, N., Vanderspek, R., Greiner, J., Castro-Tirado, A. J., Gorosabel, J., Von Hippel, T., Lehnert, M., Kuijken, K., Hoekstra, H., Metcalfe, N., Howk, C., Conselice, C., Telting, J., Rutten, R. G. M., Rhoads, J., Cole, A., Pisano, D. J., Naber, R., \& Schwarz, R. 1998, ApJ, 493, L27

Heise, J., in 't Zand, J., Kippen, M., \& Woods, P. 2001, in GRBs in the Afterglow Era, ed. E. Costa, F. Frontera, \& J. Hjorth (ESO-Springer), 16-21

Hogg, D. et al. 1997, MNRAS, 288, 404

Hurley, K., Feroci, M., Preger, B., Cline, T., \& Mazets, E. 2000, GCN notice 543

Im, M. I., Casertano, S., Griffiths, R. E., Ratnatunga, K. U., \& Tyson, J. A. 1995, ApJ, 441, 494

in' t Zand, J. . J. M., Kuiper, L., Amati, L., Antonelli, L. . A., Butler, R. . C., Costa, E., Feroci, M., Frontera, F., Gandolfi, G., Guidorzi, C., Heise, J., Kaptein, R. . G., Kuulkers, E., Nicastro, L., Piro, L., Soffitta, P., \& Tavani, M. 2001, ApJ, 559, 710

Jaunsen, A. O., Andersen, M. I., Hjorth, J., Fynbo, J. P. U., Holland, S., Thomsen, B., Gorosabel, J., Schaefer, B., Bjornsson, G., Natarajan, P., \& Tanvir, N. 2002, A\&A, submitted. astro-ph/0204278

Kennicutt, R. C. 1998, Ann. Rev. Astr. Ap., 36, 131

Kippen, R. M. et al. 2000, GCN notice 549

Koo, D. C. \& Kron, R. G. 1992, Ann. Rev. Astr. Ap., 30, 613

Kulkarni, S. R., Frail, D. A., Sari, R., Moriarty-Schieven, G. H., Shepherd, D. S., Udomprasert, P., Readhead, A. C. S., Bloom, J. S., Feroci, M., \& Costa, E. 1999, ApJ, 522, L97 
Lamb, D. Q. 2001, in GRBs in the Afterglow Era, ed. E. Costa, F. Frontera, \& J. Hjorth (ESO-Springer), 297-299

Lamb, D. Q. \& Reichart, D. E. 2000, ApJ, 536, 1

Lazzati, D., Covino, S., \& Ghisellini, G. 2002, MNRAS, 330, 583

Lazzati, D. \& Perna, R. 2002, MNRAS, 330, 383

Madau, P. 1995, ApJ, 441, 18

Nummelin, A. et al. 2000, ApJS, 128, 213

Odewahn, S. C., Djorgovski, S. G., Kulkarni, S. R., Frail, D. A., Herter, T., Fang, F., Xu, X., Pevunova, O., Steidel, C., Adelberger, K., Kellog, M., Stanek, K. Z., Garcia, M. R., \& Krockenberger, M. 1997, IAU Circ, 6735

Odewhan, S. C., Windhorst, R. A., Driver, S. P., \& Kee, W. C. 1996, ApJ, 472, L13

Paczyński, B. 1998, ApJ, 494, L45

Paerels, F., Kuulkers, E., Heise, J., \& Liedahl, D. A. 2000, ApJ, 535, L25

Panaitescu, A. \& Kumar, P. 2001, ApJ, 554, 667

Perna, R. \& Loeb, A. 1998, ApJ, 501, 467

Pian, E., Soffitta, P., Alessi, A., Amati, L., Costa, E., Frontera, F., Fruchter, A., Masetti, N., Palazzi, E., Panaitescu, A., \& Kumar, P. 2001, A\&A, 372, 456

Piran, T., Kumar, P., Panaitescu, A., \& Piro, L. 2001, ApJ, 560, L167

Piro, L. 2001, in GRBs in the Afterglow Era, ed. E. Costa, F. Frontera, \& J. Hjorth (ESOSpringer), 97-105

Piro, L., Garmire, G., Garcia, M., Stratta, G., Costa, E., Feroci, M., Meszaros, P., Vietri, M., Bradt, H., Frail, D., Frontera, F., Halpern, J., Heise, J., Hurley, K., Kawai, N., Kippen, R. M., Marshall, F., Murakami, T., Sokolov, V. V., Takeshima, T., \& Yoshida, A. 2000, Science, 290, 955

Piro, L., Garmire, G., Garcia, M. R., Antonelli, L. A., Costa, E., Feroci, M., Frail, D. A., Harrison, F., Hurley, K., Mészáros, P., \& Waxman, E. 2001, ApJ, 558, 442

Piro, L. et al. 1999, ApJ, 514, L73 
Predehl, P. \& Schmitt, J. H. M. M. 1995, A\&A, 293, 889

Reichart, D. E. 2001, ApJ, 553, 235

Reichart, D. E. 2001, ApJ, submitted; astro-ph/0107546

Reichart, D. E. \& Price, P. A. 2002, ApJ, 565, 174

Reichart, D. E. \& Yost, S. A. 2001, ApJ, submitted; astro-ph/0107545

Sari, R. \& Piran, T. 1999, ApJ, 520, 641

Sari, R., Piran, T., \& Narayan, R. 1998, ApJ, 497, L17

Schechter, P. 1976, ApJ, 203, 297

Solomon, P. M., Rivolo, A. R., Barrett, J., \& Yahil, A. 1987, ApJ, 319, 730

Stornelli, M. et al. 2000, GCN notice 540

Taylor, G. B., Bloom, J. S., Frail, D. A., Kulkarni, S. R., Djorgovski, S. G., \& Jacoby, B. A. 2000, ApJ, 537, L17

Ward-Thompson, D., Scott, P. F., Hills, R. E., \& Andre, P. 1994, MNRAS, 268, 276

Waxman, E. \& Draine, B. T. 2000, ApJ, 537, 796

Waxman, E., Kulkarni, S. R., \& Frail, D. A. 1998, ApJ, 497, 288

Yoshida, A., Yonetoku, N. M., Murakami, T., Otani, C., Kawai, N., Ueda, Y., Shibata, R., \& Uno, S. 2001, ApJ, 557, L27 


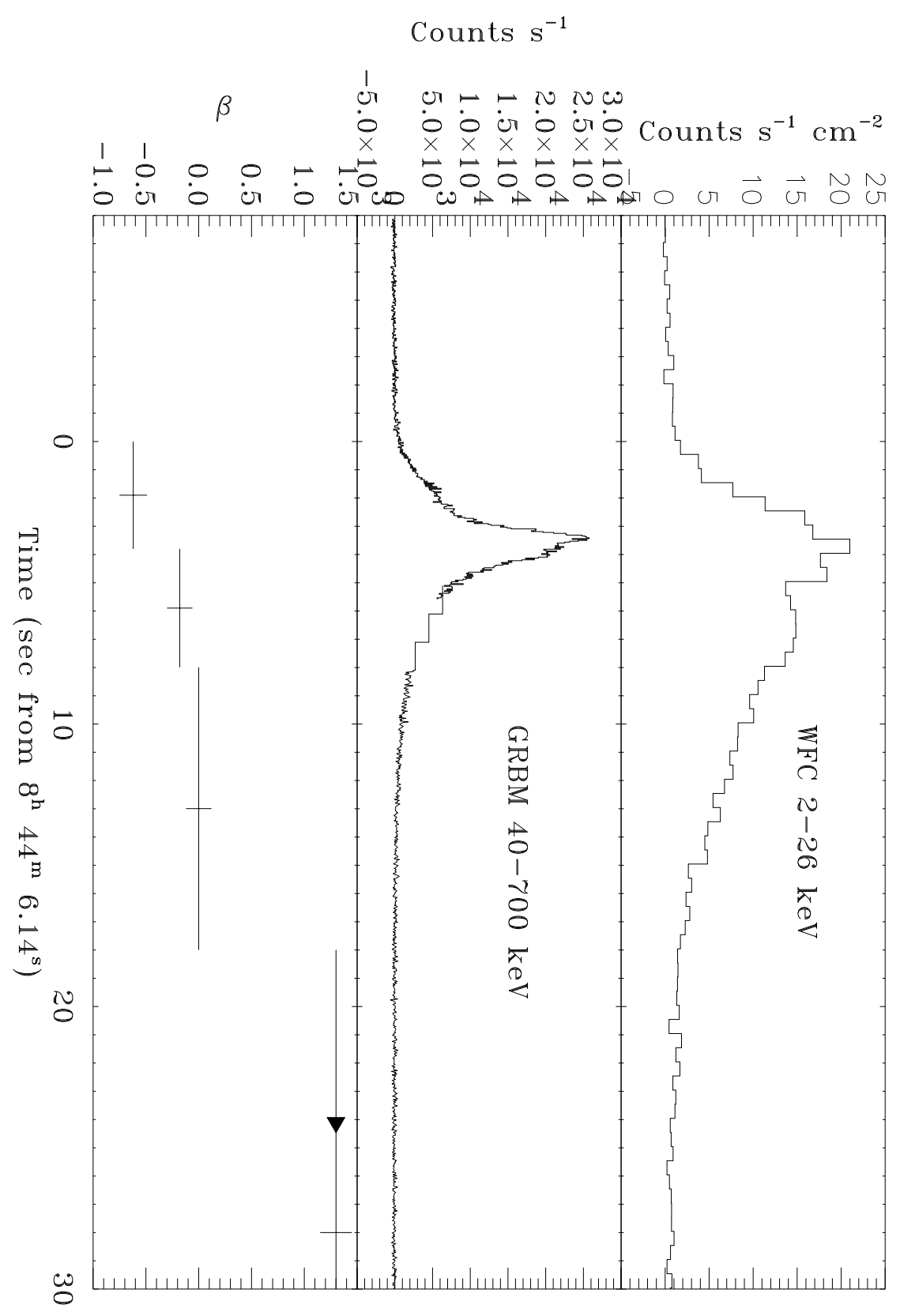

Fig. 1. - Light curves of GRB 000210 in the BeppoSAX WFC (upper panel), GRBM (middle panel) and energy spectral index $\left(\beta=\Gamma-1, F \propto E^{-\beta}\right)$ evolution in the WFC (lower panel). The gap between 5.5 and $8 \mathrm{sec}$ in the GRBM data is due to a telemetry loss. In that interval we have plotted the $1 \mathrm{sec}$ resolution data of the ratemeter of the instrument, while the other data points have a time bin of $31.25 \mathrm{~ms}$. The last point of the spectral index is relative to the interval $18-80 \mathrm{~s}$. 


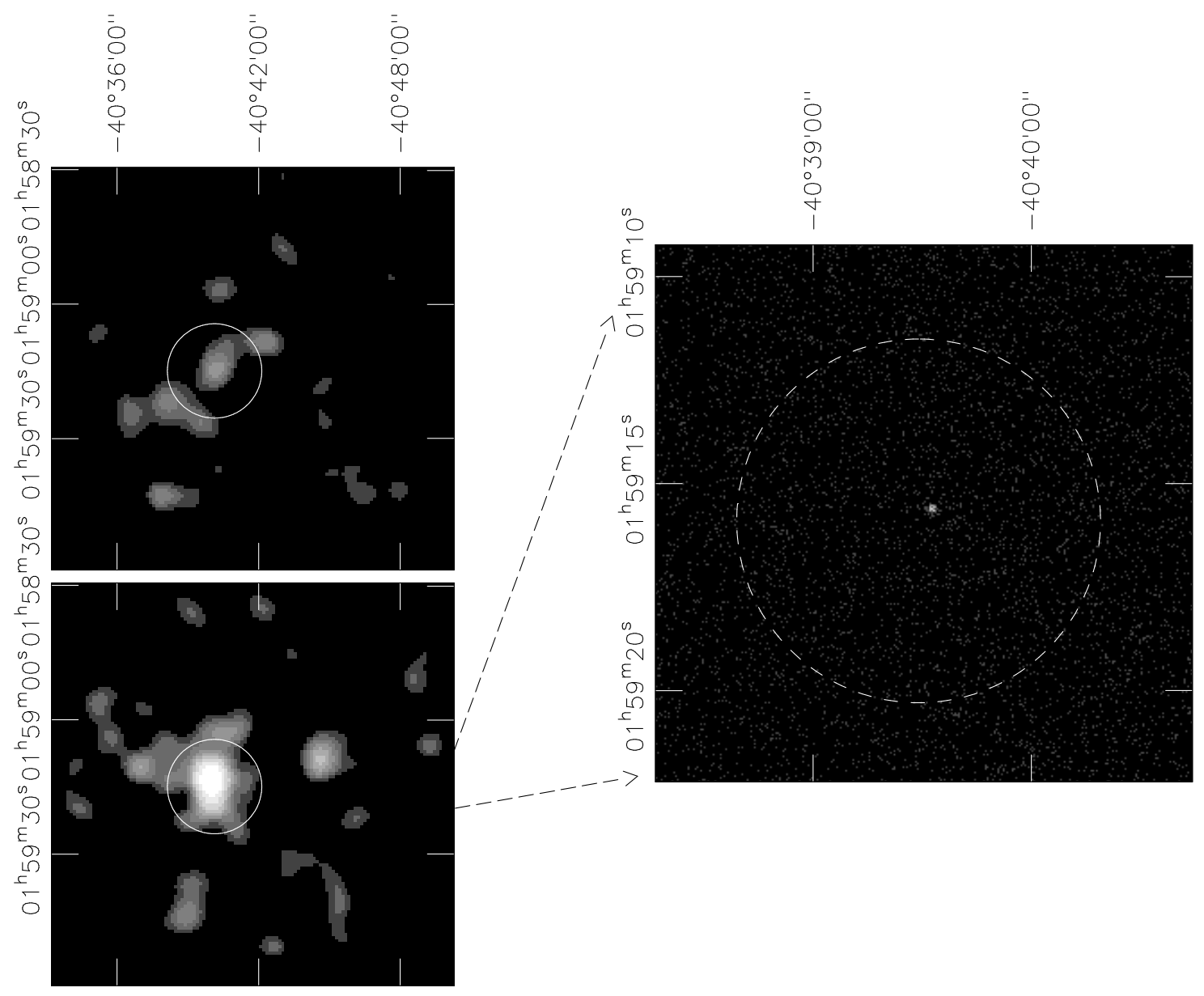

Fig. 2.- Image of the X-ray afterglow of GRB 000210 by BeppoSAX and Chandra. Upper panel: the left and right images show the afterglow in the BeppoSAX MECS(1.6-10 keV) 8 hrs and $30 \mathrm{hrs}$ after the GRB respectively. The circle represents the WFC error box. Lower panel: the Chandra ACIS-S(0.2-8 keV) image of the afterglow $21 \mathrm{hrs}$ after the GRB. The dashed circle is the BeppoSAX NFI error box 


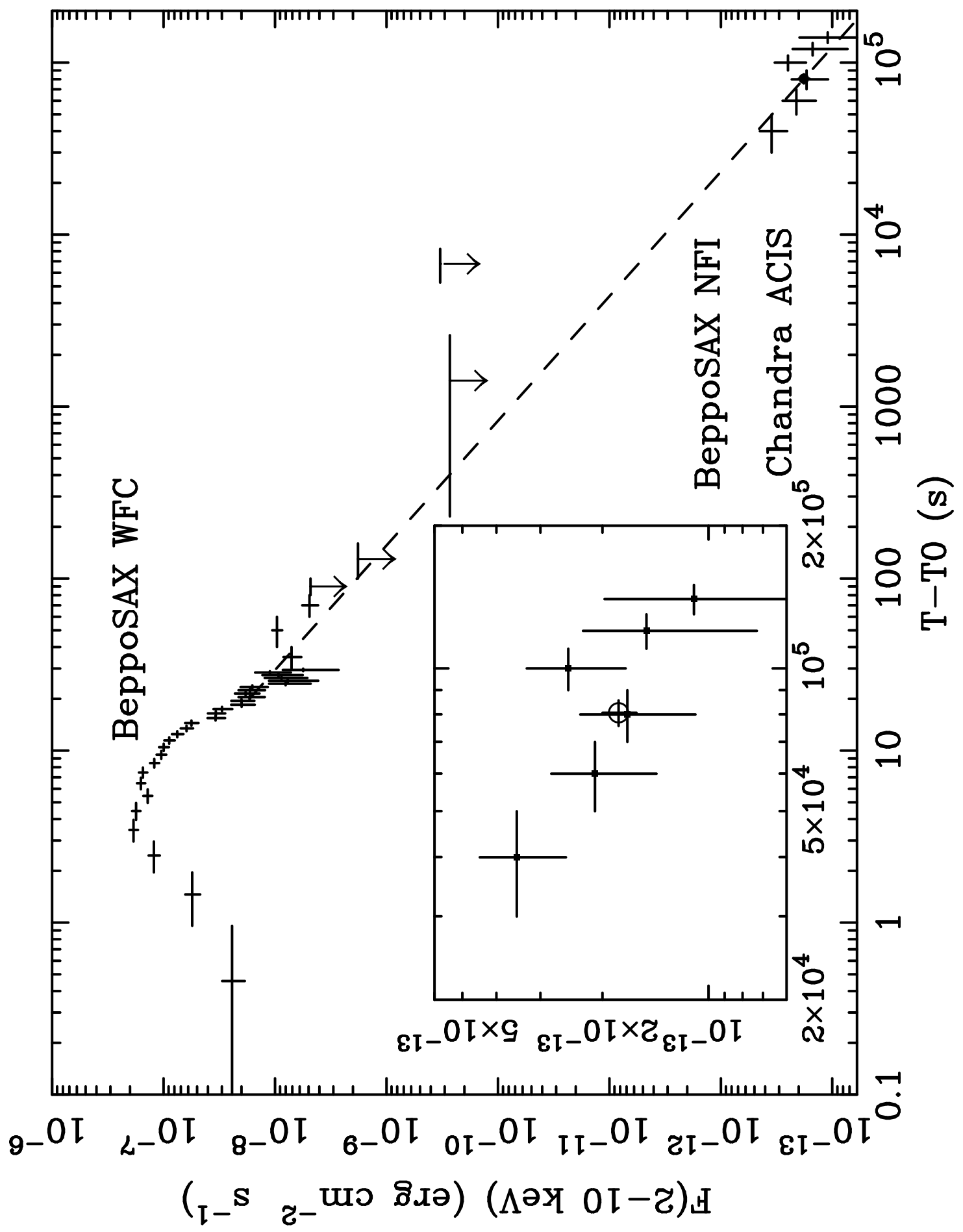

Fig. 3.- Light curve from BeppoSAX WFC (from 0.1 to 5000 s), and BeppoSAX NFI and Chandra ACIS (from 30.000 to $140.000 \mathrm{~s}$ ). The latter data are expanded in the inset. The Chandra data point is identified by an open circle 


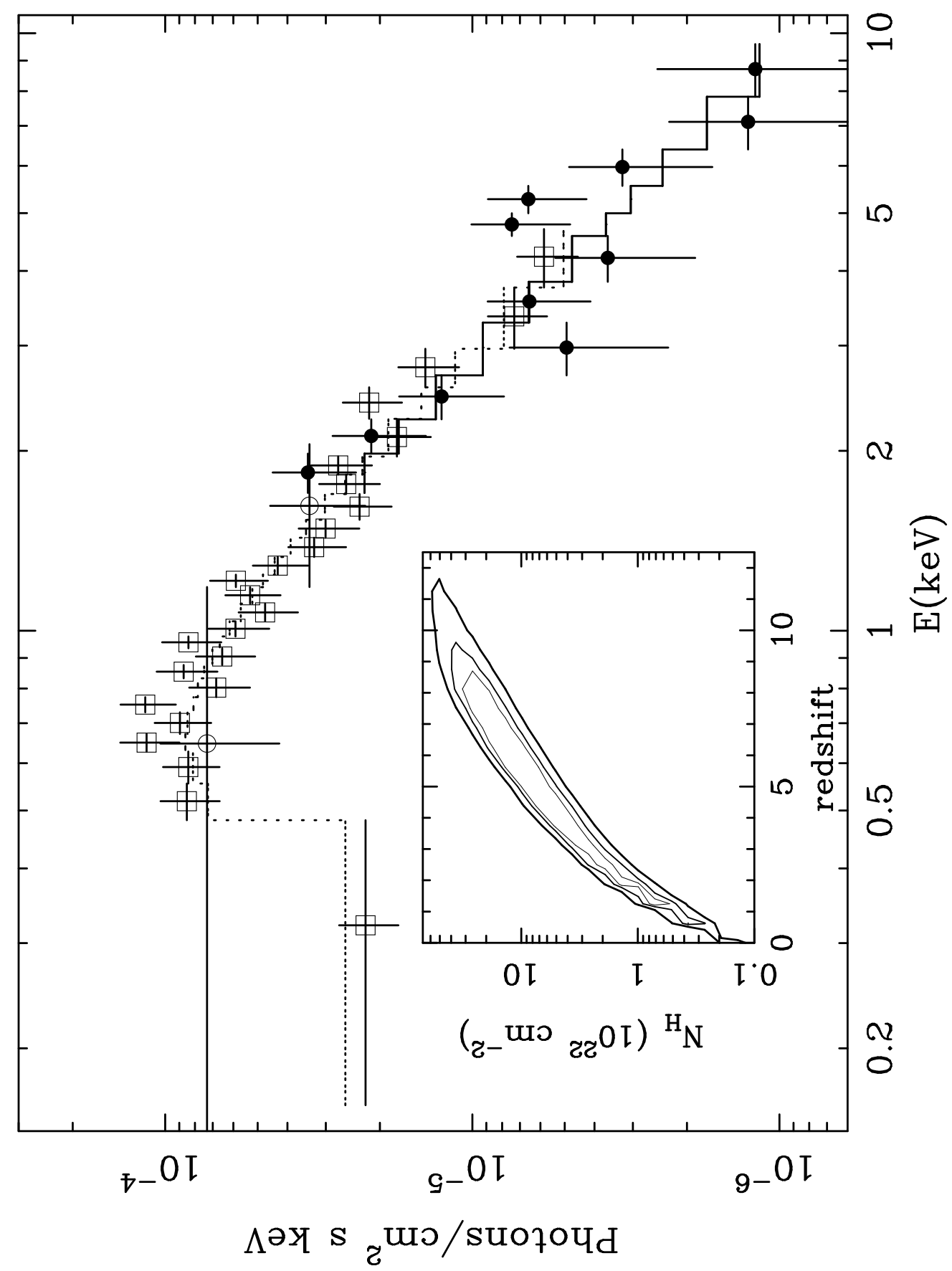

Fig. 4.- The X-ray spectrum of the afterglow by BeppoSAX LECS (empty circles), MECS (filled circles) \& Chandra ACIS-S (empty squares). The continuous (dashed) line is the best fit absorbed power law to the BeppoSAX (Chandra) data. The contour plot of the intrinsic absorption column density as a function of the redshift is plotted in the inset. The contours correspond to $68 \%, 90 \%$ and $99 \%$ confidence level (thin, normal, thick lines) 


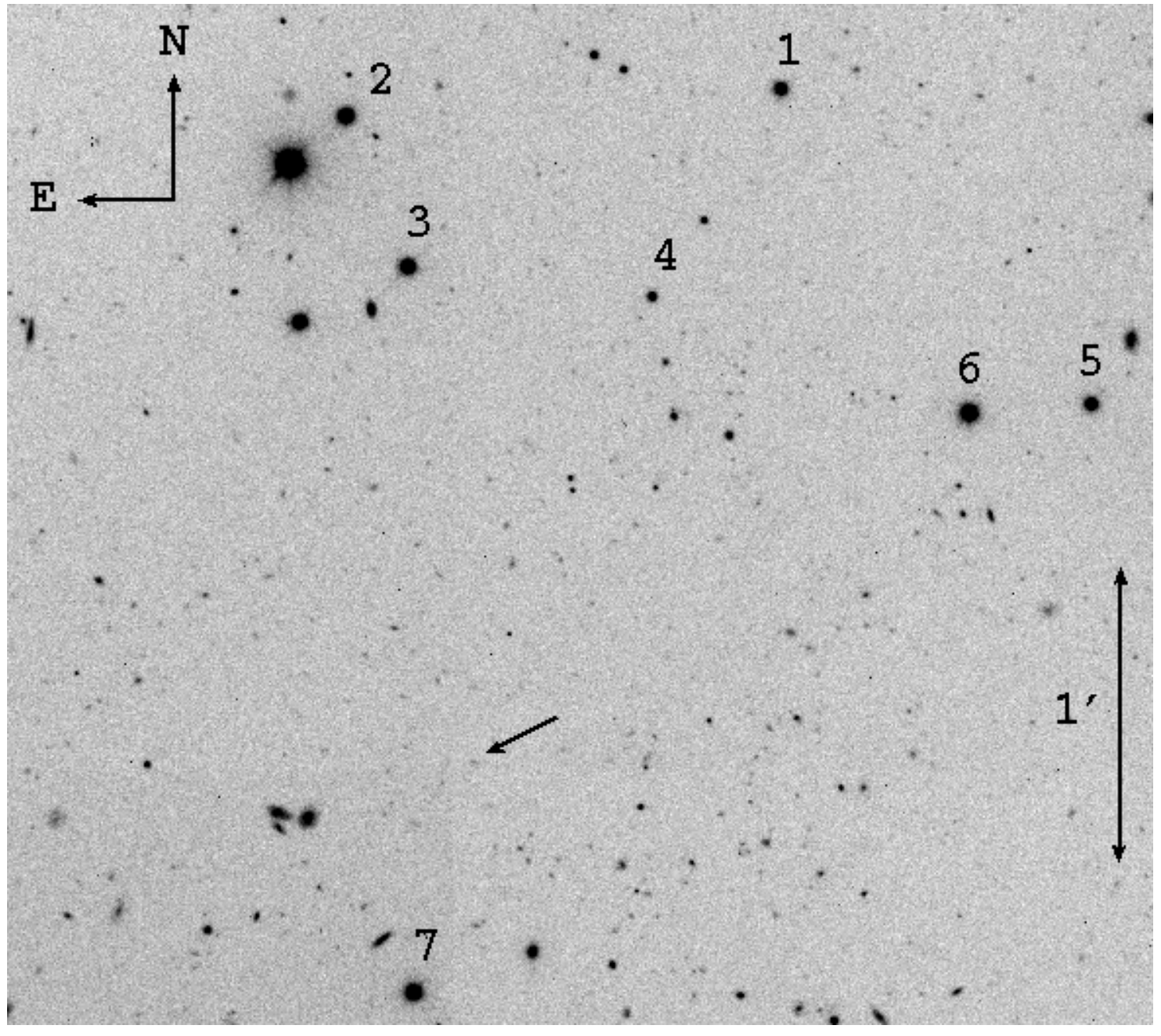

Fig. 5.- R-band VLT image of the GRB000210 field. The numbers label the secondary standards (Tab. 2) 


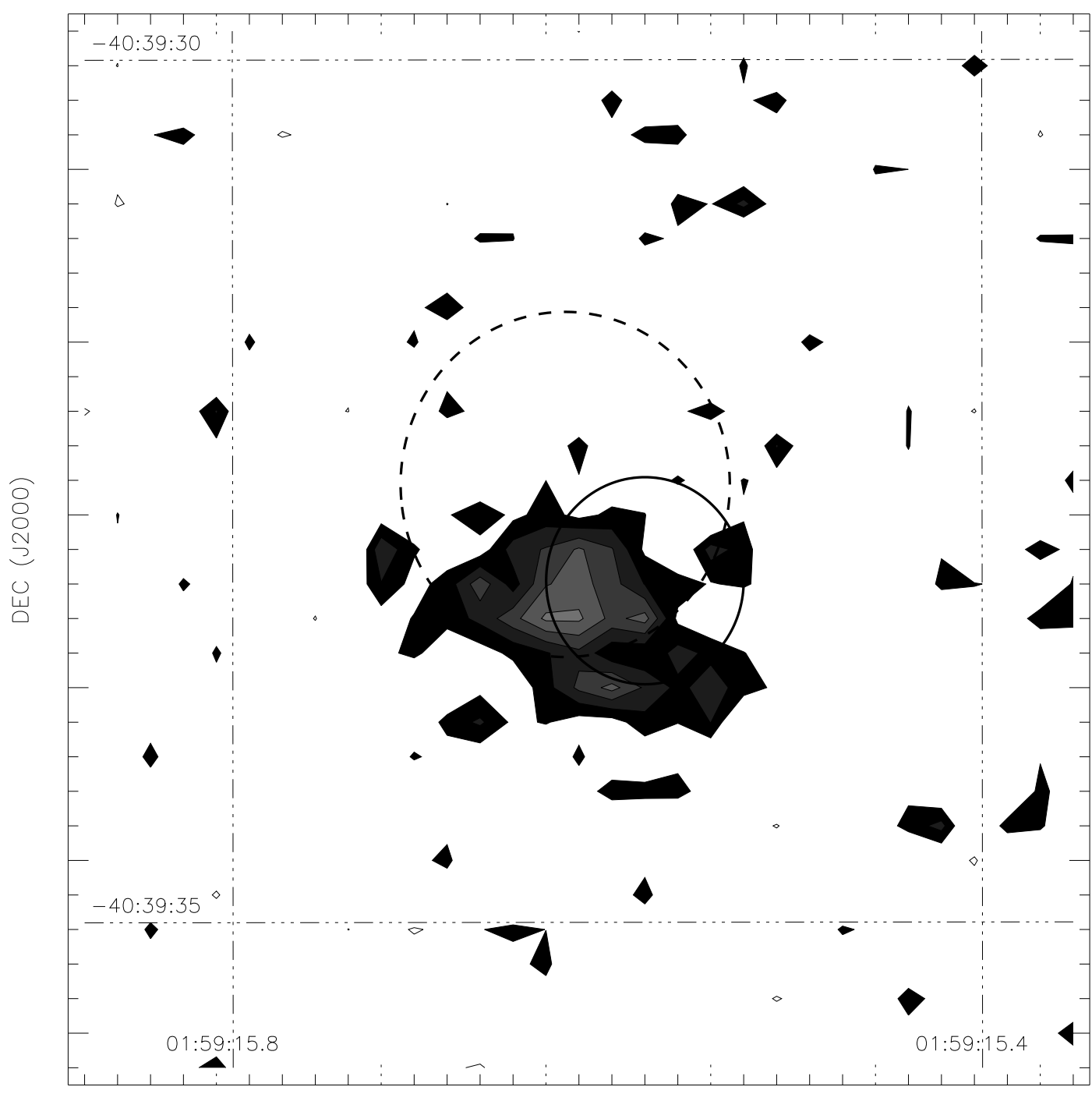

RA (J2000)

Fig. 6. - Blow-up of previous figure, showing the contour plot of the likely host galaxy of GRB000210. The circles show the 90\% error circles of the Chandra (continuous line) and radio (dashed line) afterglows. 


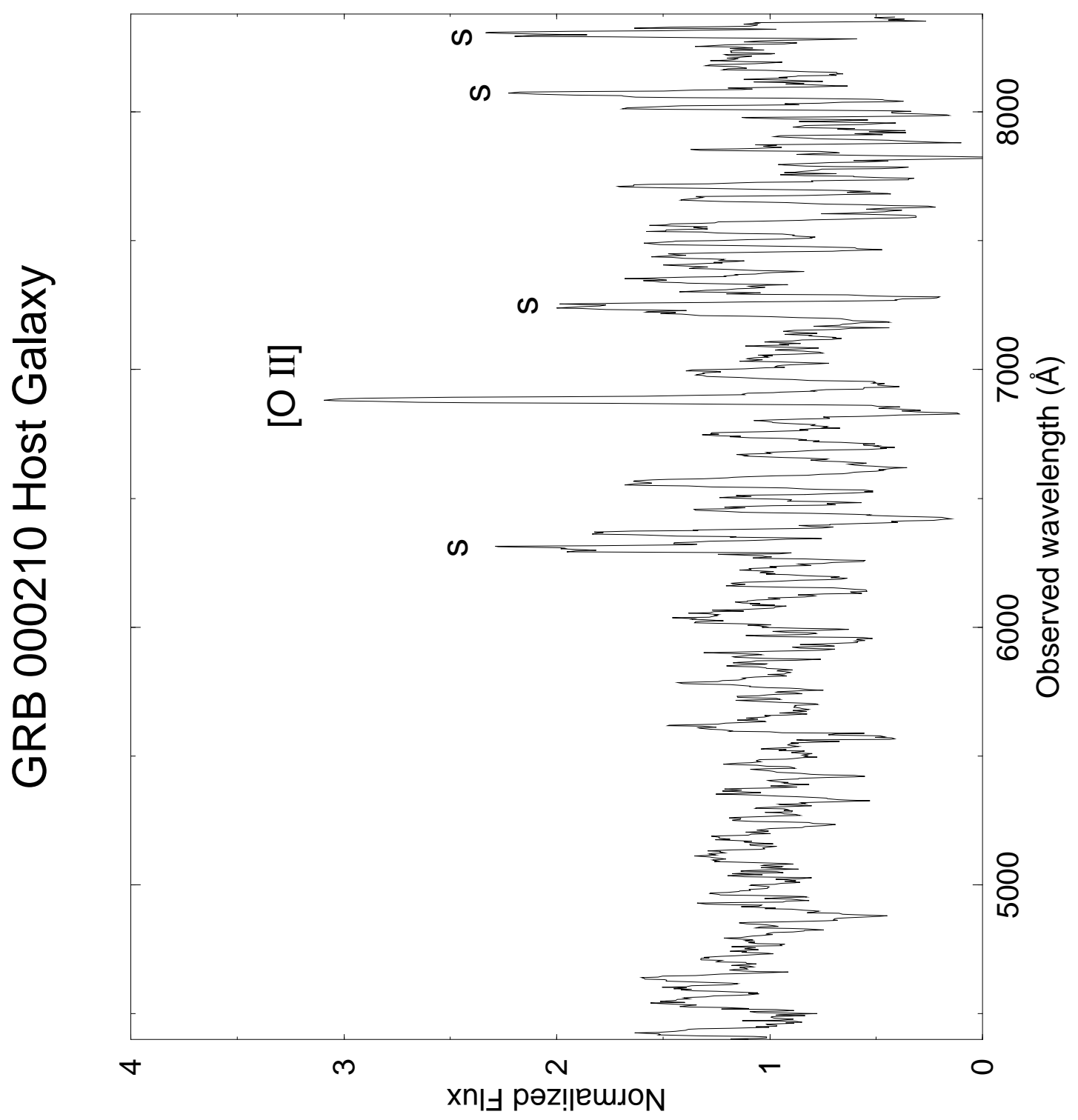

Fig. 7.- The normalized spectrum of the GRB 000210 host galaxy acquired with VLT+FORS1. The spectrum has been smoothed with a boxcar width corresponding to the instrumental spectral resolution $(10 \AA)$. The spikes, mostly present in the red part of the spectrum and indicated with an $\mathrm{S}$, are residuals from the subtraction of strong sky emission lines. The region of the [OII] line is not affected by sky emission lines. 
Table 1. Optical Observations of GRB 000210

\begin{tabular}{|c|c|c|c|c|c|}
\hline $\begin{array}{c}\text { Epoch } \\
\text { (UT) }\end{array}$ & Filter & $\begin{array}{l}\text { Exposure } \\
\text { Time (s) }\end{array}$ & $\begin{array}{l}\text { Seeing } \\
(\text { arcsec) }\end{array}$ & Telescope & Magnitude \\
\hline Feb. $10.88-10.90$ & $\mathrm{R}$ & $3 \times 300$ & 1.2 & NOT & $>23$ \\
\hline Feb. $11.03-11.08$ & $\mathrm{R}$ & $10 \times 300$ & 1.6 & $1.54 \mathrm{D}$ & $23.5 \pm 0.2$ \\
\hline Feb. $14.02-14.03$ & $\mathrm{R}$ & 600 & 1.9 & $1.54 \mathrm{D}$ & $>22.6$ \\
\hline May $5.42-5.44$ & $\mathrm{R}$ & $2 \times 600$ & 2.3 & $1.54 \mathrm{D}$ & $>22$ \\
\hline Aug. $22.29-22.41$ & $\mathrm{R}$ & $7 \times 900$ & 2.2 & $1.54 \mathrm{D}$ & $23.47 \pm 0.10$ \\
\hline Aug. $23.23-23.29$ & $\mathrm{R}$ & $5 \times 900$ & 2.3 & $1.54 \mathrm{D}$ & $"$ \\
\hline Aug. $24.23-24.30$ & $\mathrm{R}$ & $4 \times 900$ & 3.0 & $1.54 \mathrm{D}$ & $"$ \\
\hline Aug. $26.29-26.43$ & $\mathrm{~V}$ & $9 \times 900$ & 1.5 & $1.54 \mathrm{D}$ & $24.09 \pm 0.15$ \\
\hline Aug. $27.21-27.24$ & $\mathrm{I}$ & $2 \times 900$ & 1.4 & $1.54 \mathrm{D}$ & $22.60 \pm 0.12$ \\
\hline Aug. $28.21-28.24$ & I & $2 \times 1200$ & 1.4 & $1.54 \mathrm{D}$ & $"$ \\
\hline Aug. $29.21-29.30$ & $\mathrm{I}$ & $7 \times 1200$ & 1.1 & $1.54 \mathrm{D}$ & $"$ \\
\hline Aug. $30.22-30.24$ & I & 1200 & 1.1 & $1.54 \mathrm{D}$ & $"$ \\
\hline Aug. $31.21-31.24$ & $\mathrm{~B}$ & $2 \times 1200$ & 1.5 & $1.54 \mathrm{D}$ & $25.1 \pm 0.7$ \\
\hline Oct. $25.24-25.24$ & $\mathrm{R}$ & 300 & 0.7 & VLT & $23.46 \pm 0.10$ \\
\hline
\end{tabular}

Note. - R and I magnitudes from Aug. 22 to Aug. 24 and from Aug.27 to Aug.30 have been derived by summing the images obtained in those nights 
Table 2. Positions and magnitudes of the secondary standards reported in Fig.5

\begin{tabular}{cccccccc}
\hline \hline ID & $\alpha_{2000}$ & $\delta_{2000}$ & $\mathrm{I}$ & $\mathrm{R}$ & $\mathrm{V}$ & $\mathrm{B}$ & $\mathrm{U}$ \\
\hline 1 & $01: 59: 10.056$ & $-40: 37: 15.11$ & $17.21 \pm 0.02$ & $17.71 \pm 0.02$ & $18.20 \pm 0.02$ & $19.01 \pm 0.03$ & $19.64 \pm 0.08$ \\
2 & $01: 59: 17.877$ & $-40: 37: 20.58$ & $16.51 \pm 0.02$ & $16.91 \pm 0.02$ & $17.26 \pm 0.02$ & $17.81 \pm 0.03$ & $17.88 \pm 0.05$ \\
3 & $01: 59: 16.762$ & $-40: 37: 51.37$ & $15.93 \pm 0.02$ & $17.00 \pm 0.02$ & $18.00 \pm 0.02$ & $19.40 \pm 0.03$ & $20.71 \pm 0.12$ \\
4 & $01: 59: 12.370$ & $-40: 37: 57.60$ & $18.09 \pm 0.03$ & $19.15 \pm 0.03$ & $20.18 \pm 0.03$ & $21.54 \pm 0.04$ & $23.11 \pm 0.25$ \\
5 & $01: 59: 04.478$ & $-40: 38: 19.70$ & $17.34 \pm 0.02$ & $17.64 \pm 0.02$ & $17.86 \pm 0.02$ & $18.16 \pm 0.03$ & $17.99 \pm 0.06$ \\
6 & $01: 59: 06.665$ & $-40: 38: 21.51$ & $16.07 \pm 0.02$ & $16.52 \pm 0.02$ & $16.89 \pm 0.02$ & $17.47 \pm 0.03$ & $17.49 \pm 0.04$ \\
7 & $01: 59: 16.673$ & $-40: 40: 20.09$ & $16.60 \pm 0.02$ & $16.74 \pm 0.02$ & $16.78 \pm 0.02$ & $16.83 \pm 0.02$ & $17.07 \pm 0.03$ \\
\hline
\end{tabular}

Table 3. Radio Observations of GRB 000210

\begin{tabular}{cccccr}
\hline \hline $\begin{array}{c}\text { Epoch } \\
\text { (UT) }\end{array}$ & $\begin{array}{c}\Delta \mathrm{t} \\
(\text { days })\end{array}$ & $\begin{array}{c}\nu \\
(\mathrm{GHz})\end{array}$ & Telescope & Array & $\begin{array}{c}\mathrm{S} \pm \sigma \\
(\mu \mathrm{Jy})\end{array}$ \\
\hline 2000 Feb. 10.98 & 0.62 & 8.46 & VLA & $\mathrm{B}$ & $34 \pm 70$ \\
2000 Feb. 12.32 & 1.96 & 8.70 & ATCA & $6 \mathrm{~A}$ & $-4 \pm 59$ \\
2000 Feb. 14.90 & 4.54 & 8.46 & VLA & $\mathrm{B}$ & $15 \pm 37$ \\
2000 Feb. 15.03 & 4.67 & 8.46 & VLA & $\mathrm{CnB}$ & $-34 \pm 42$ \\
2000 Feb. 18.95 & 8.59 & 8.46 & VLA & $\mathrm{CnB}$ & $93 \pm 21$ \\
2000 Feb. 26.95 & 16.59 & 8.46 & VLA & $\mathrm{CnB}$ & $20 \pm 16$ \\
2000 Mar. 03.92 & 21.56 & 8.46 & VLA & $\mathrm{CnB}$ & $58 \pm 33$ \\
2000 Mar. 27.81 45.45 & 8.46 & VLA & $\mathrm{C}$ & $45 \pm 45$ \\
2000 May 28.73 & 107.37 & 8.46 & VLA & $\mathrm{C}$ & $48 \pm 26$ \\
2000 Jun. 24.69 134.33 & 8.46 & VLA & $\mathrm{D}$ & $-1 \pm 34$ \\
\hline
\end{tabular}

Note. - The columns are (left to right), (1) UT date for each observation, (2) time elapsed since the $\gamma$-ray burst, (3) observing frequency, (4) telescope name, (5) the array configuration, and (6) peak flux density at the best fit position of the radio transient, with the error given as the root mean square noise in the image. 